\title{
Search for Laser-Induced Formation of Antihydrogen Atoms
}

\author{
M. Amoretti, ${ }^{1}$ C. Amsler, ${ }^{2}$ G. Bonomi, ${ }^{3,4}$ P. D. Bowe, ${ }^{5}$ C. Canali, ${ }^{1,6}$ C. Carraro, ${ }^{1,6}$ C. L. Cesar, ${ }^{7}$ M. Charlton, ${ }^{8}$ \\ A. M. Ejsing, ${ }^{5}$ A. Fontana, ${ }^{4,9}$ M. C. Fujiwara, ${ }^{10}$ R. Funakoshi, ${ }^{10}$ P. Genova, ${ }^{4,9}$ J. S. Hangst, ${ }^{5}$ R. S. Hayano, ${ }^{10}$ \\ L. V. Jørgensen ${ }^{8}$ A. Kellerbauer, ${ }^{11, *}$ V. Lagomarsino, ${ }^{1,6}$ E. Lodi Rizzini, ${ }^{12,13}$ M. Macrì, ${ }^{1}$ N. Madsen, ${ }^{5}$ G. Manuzio, ${ }^{1,6}$ \\ D. Mitchard, ${ }^{8}$ P. Montagna, ${ }^{4,9}$ L. G. C. Posada,${ }^{10}$ H. Pruys,${ }^{2}$ C. Regenfus, ${ }^{2}$ A. Rotondi, ${ }^{4,9}$ H. H. Telle, ${ }^{8}$ G. Testera, ${ }^{1}$ \\ D. P. Van der Werf, ${ }^{8}$ A. Variola, ${ }^{1}$ L. Venturelli, ${ }^{12,13}$ Y. Yamazaki, ${ }^{14}$ and N. Zurlo ${ }^{12,13}$
}

(ATHENA Collaboration)

\author{
${ }^{1}$ Istituto Nazionale di Fisica Nucleare, Sezione di Genova, 16146 Genova, Italy \\ ${ }^{2}$ Physik-Institut, Zürich University, 8057 Zürich, Switzerland \\ ${ }^{3}$ Dipartimento di Ingegneria Meccanica, Università di Brescia, 25123 Brescia, Italy \\ ${ }^{4}$ Istituto Nazionale di Fisica Nucleare, Università di Pavia, 27100 Pavia, Italy \\ ${ }^{5}$ Department of Physics and Astronomy, University of Aarhus, 8000 Aarhus C, Denmark \\ ${ }^{6}$ Dipartimento di Fisica, Università di Genova, 16146 Genova, Italy \\ ${ }^{7}$ Instituto de Fisica, Univesidade Federal do Rio de Janeiro, Rio de Janeiro 21945-970, Brazil \\ ${ }^{8}$ Department of Physics, University of Wales Swansea, Swansea SA2 8PP, United Kingdom \\ ${ }^{9}$ Dipartimento di Fisica Nucleare e Teorica, Università di Pavia, 27100, Pavia, Italy \\ ${ }^{10}$ Department of Physics, University of Tokyo, Tokyo 113-0033, Japan \\ ${ }^{11}$ Physics Department, CERN, 1211 Geneva 23, Switzerland \\ ${ }^{12}$ Dipartimento di Chimica e Fisica per l'Ingegneria e per i Materiali, Università di Brescia, 25123 Brescia, Italy \\ ${ }^{13}$ Istituto Nazionale di Fisica Nucleare, Gruppo Collegato di Brescia, 25123 Brescia, Italy \\ ${ }^{14}$ Atomic Physics Laboratory, RIKEN, Saitama 351-0198, Japan
}

(Received 14 July 2006; published 22 November 2006)

\begin{abstract}
Antihydrogen can be synthesized by mixing antiprotons and positrons in a Penning trap environment. Here an experiment to stimulate the formation of antihydrogen in the $n=11$ quantum state by the introduction of light from a $\mathrm{CO}_{2}$ continuous wave laser is described. An overall upper limit of $0.8 \%$ with $90 \%$ C.L. on the laser-induced enhancement of the recombination has been found. This result strongly suggests that radiative recombination contributes negligibly to the antihydrogen formed in the experimental conditions used by the ATHENA Collaboration.
\end{abstract}

DOI: $10.1103 /$ PhysRevLett.97.213401

PACS numbers: $36.10 .-\mathrm{k}, 25.43 .+\mathrm{t}$

In 2002, two experiments at CERN, first ATHENA [1] and then ATRAP [2], reported the production of cold antihydrogen $(\overline{\mathrm{H}})$ by mixing antiprotons and positrons at low temperature in a nested Penning trap [3]. The ultimate goal of these experiments is to test matter-antimatter $(C P T)$ symmetry by means of precision two-photon spectroscopy of the $\overline{\mathrm{H}} 1 S-2 S$ transition, and, hence, the development of methods to enhance the production of cold, ground-state $\overline{\mathrm{H}}$ atoms is of great importance.

In this Letter, we report the result of the first such attempt, i.e., to stimulate the formation of $\overline{\mathrm{H}}$ in the $n=$ 11 quantum state (which would radiatively deexcite to the ground state) by introducing $\lambda=11 \mu \mathrm{m}$ laser light from a $\mathrm{CO}_{2}$ laser into the ATHENA apparatus [4]. In Ref. [1], we used antiprotons delivered by CERN's Antiproton Decelerator and a low energy positron beam derived from a ${ }^{22} \mathrm{Na}$ radioactive source $\left(1.4 \times 10^{9} \mathrm{~Bq}\right)$. Both the $\bar{p}$ and the $e^{+}$were trapped, cooled, and accumulated in separate traps prior to moving, and subsequently mixing, them in a common trap (called the mixing trap). The positron accumulation trap was located inside a room temperature vacuum chamber in a $0.14 \mathrm{~T}$ magnetic field. The antiproton capture trap and the mixing trap were located in the $3 \mathrm{~T}$ field of a superconducting magnet, where the temperature was maintained at about $15 \mathrm{~K}$, in ultrahigh vacuum conditions.

The detector surrounding the mixing trap allows the different events which occur after mixing to be fully identified through the decay products of the annihilations (charged mesons from $\bar{p}, \gamma$ from $e^{+}$), making possible the vertex reconstruction of antiproton and positron annihilations $[5,6]$.

In the "standard mixing cycle," the mixing trap, configured as a nested Penning trap [3], allows the simultaneous trapping of oppositely charged particles. The central part of the trap is filled with 3-7 $\times 10^{7} e^{+}$. Once these have self-cooled by emission of synchrotron radiation, about $10^{4} \bar{p}$ are injected and the two particle species interact for around $1 \mathrm{~min}$. At the start of each mixing cycle, the antiprotons are cooled by their passage through the positron plasma, and after a few tens of milliseconds $\overline{\mathrm{H}}$ formation begins $[7,8]$. At the end of the mixing cycle, the 
nested trap is emptied and the number of both positrons and antiprotons are counted before the process is restarted.

In so-called cold mixing, when the positron temperature is that of the trap at $15 \mathrm{~K}$, most of the annihilations come from $\overline{\mathrm{H}}$ atoms that escape and annihilate on the trap walls; the background is due to a small fraction of antiprotons annihilating near the trap center on residual gas atoms or ions.

In the hot mixing cycle, when the positron plasma is heated by exciting its axial dipole resonance (around $20 \mathrm{MHz}$ [9]), $\overline{\mathrm{H}}$ production is observed to decrease with temperature [8]; when the positron plasma is heated up to $8000 \mathrm{~K}$, only background events are observed $[1,8]$.

These mixing experiments may be analyzed with the knowledge that the two main processes expected to result in $\overline{\mathrm{H}}$ formation are the two-body radiative capture $\left(e^{+}+\right.$ $\bar{p} \rightarrow \overline{\mathrm{H}}+\gamma)$ and three-body combination $\left(e^{+}+e^{+}+\right.$ $\bar{p} \rightarrow \overline{\mathrm{H}}+e^{+}$) [10].

Given a temperature of $15 \mathrm{~K}$, and assuming complete overlap between the two particle clouds (formed by $10^{4} \bar{p}$ and a positron plasma of density $1.7 \times 10^{8} \mathrm{~cm}^{-3}$ ), the expected two-body radiative $\overline{\mathrm{H}}$ production rate at the beginning of the mixing is about $40 \mathrm{~s}^{-1}$, an order of magnitude lower than our measured value of $432 \pm 44 \mathrm{~s}^{-1}$ [8]. Thus, the measured production rate is not obviously compatible with a simple radiative calculation assuming thermal equilibrium.

On the other hand, the naive scaling for the three-body reaction, $T^{-9 / 2}[10,11]$, is clearly inconsistent with our data [8]. However, simulations of three-body capture in a nested trap have shown that this reaction is a multistep process that strongly depends on the plasma conditions and on the trap dynamics, so that collisional relaxation and the finite transit time of the antiprotons through the positron plasma give a shallower temperature scaling $[12,13]$.

In an effort to clarify the situation, we have investigated the radiative $\overline{\mathrm{H}}$ formation mechanism by attempting to stimulate capture using laser light. Laser-stimulated electron-proton recombination for the conditions encountered in ion storage rings was first examined in Ref. [14], and the first observations of laser-induced recombination obtained with merged beams of protons and electrons were reported in Refs. [15,16]. In a steady state situation, the laser-mediated electron-proton recombination rate is given by [17]

$$
R(E)_{n, l}=N_{p} N(E) w_{n l} \frac{r_{n, l}^{\mathrm{abs}}(E) \gamma_{n, l}}{r_{n, l}^{\mathrm{abs}}(E)+\gamma_{n, l}},
$$

where $E$ is electron energy, $N(E)$ is the level population function of a continuum Coulomb state according to the positron density and the Maxwell-Boltzmann distribution, $w_{n l}$ is the statistical weight of the lower level $(n, l), N_{p}$ is the number of protons, $r_{n, l}^{\text {abs }}$ is the induced radiation absorption rate, and $\gamma_{n, l}$ is the spontaneous radiative decay rate of the level $(n, l)$. The absorption rate $r_{n, l}^{\text {abs }}$ is inversely proportional to the energy difference between the continuum and level $(n, l)$ and, thus, inversely proportional to the stimulating photon energy. Thus, Eq. (1) suggests that high power densities and small laser photon energies should promote high enhancement factors. In effect, electron-proton-stimulated recombination with a laser on/ off ratio $\simeq 4$ has been observed in a single-pass merged beam experiment with a $15 \mathrm{~W}$ continuously operating $\mathrm{CO}_{2}$ laser beam $(\lambda=10.5 \mu \mathrm{m})$ crossing the interaction region [16].

This type of technique has been implemented by ATHENA, using a $\mathrm{CO}_{2}$ laser to stimulate the radiative formation of $\overline{\mathrm{H}}$ in the quantum state with $n=11$. The transition originates in the continuum, and therefore the recombination rate is affected neither by the (finite) Doppler profile width prevalent at $T=15 \mathrm{~K}$ nor by the laser bandwidth $\left(\Delta \nu_{L} \simeq 100 \mathrm{MHz}\right)$; this is because the level population and oscillator strength are nearly constant within these widths.

The laser-stimulated positron-antiproton recombination process can be described by an equation analogous to Eq. (1), but now using the number of antiprotons and positrons. Therefore, summing over all the accessible final states in Eq. (1), considering the Doppler width, the laser bandwidth, the effect of the $3 \mathrm{~T}$ magnetic field (for the Zeeman splitting of the energy levels in the PaschenBack regime), and a laser power of $100 \mathrm{~W} \mathrm{~cm}^{-2}(\lambda=$ $10.96 \mu \mathrm{m}$ ), and assuming a complete overlap between $10^{4}$ antiprotons and a positron plasma of $10^{8} e^{+} \mathrm{cm}^{-3}$, we can calculate the expected stimulated formation rate for our laser runs. We obtain a rate of about $60 \overline{\mathrm{H}}$ atoms s ${ }^{-1}$ under equilibrium conditions at $15 \mathrm{~K}$. The saturation power density is of the order of $1000 \mathrm{~W} \mathrm{~cm}^{-2}$. Since at this positron plasma density the expected spontaneous radiative transition rate is $\simeq 24 \mathrm{~s}^{-1}$, the predicted enhancement between laser on and laser off is about $(60+24) / 24=$ 3.5. The antiproton motion in the axial direction in and out of the $e^{+}$cloud has not been taken into account. However, at the energies (relative to the $e^{+}$) at which $\overline{\mathrm{H}}$ is formed [7], the time spent in the lateral wells of the nested trap is negligible, since the $\bar{p}$ have an additional kinetic energy of up to $\simeq 15 \mathrm{eV}$ there.

The previous estimate does not consider nonradiative collisional recombination processes, which in our case seem to be dominant (at least $90 \%$ at the beginning of the mixing cycle [8]). We note also that collisional formation favors high- $n$ states which can be ionized by the laser. This effect is proportional to the flight time of the formed $\overline{\mathrm{H}}$ in the laser light and on relaxation properties of the final state, i.e., by the spontaneous radiative decay to lower levels not influenced by the laser. If we consider the spontaneous radiative decay of states and assume that the $\overline{\mathrm{H}}$ atoms are formed in equilibrium with the $15 \mathrm{~K}$ positron plasma (flight time of about $1 \mu \mathrm{s}$ in the laser light), we can calculate the behavior of the ionization rate as a function of the principal quantum number. We find, for the 11D state, a radiative rate of $1.37 \mu \mathrm{s}^{-1}$ and an ionization rate of $0.86 \mu \mathrm{s}^{-1}$. For $11<n<60$, the ionization rate is always 


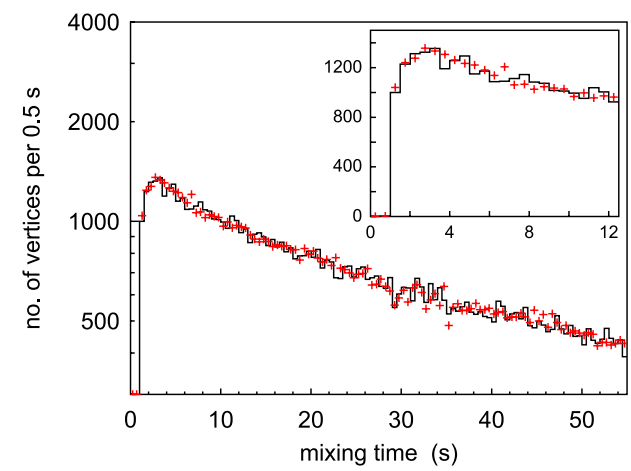

FIG. 1 (color online). Vertex time distribution (log scale) for laser off (line) and laser on (crosses). Inset: The same for the first $12 \mathrm{~s}$ in decimal scale.

lower than the radiative one. For example, for a $\overline{\mathrm{H}}$ atom in the $20 P$ and $20 D$ states, we find ionization probabilities of $35 \%$ and $22 \%$, respectively. Since in our case the temperature of the formed $\overline{\mathrm{H}}$ atoms is higher than the temperature of the positron plasma [18], we estimate an interaction time of $100 \mathrm{~ns}$, resulting in ionization probabilities 10 times lower. Nevertheless, there is a finite probability for the laser to ionize some Rydberg states; however, a quantitative estimation cannot be made without detailed knowledge of the $\overline{\mathrm{H}}$ velocity and quantum state distributions, which is currently not available.

The ATHENA experimental apparatus has been modified to allow the insertion of laser light into the mixing trap. A $\mathrm{CO}_{2}$ continuous wave laser was used with a tunable wavelength of $9.5<\lambda<11.2 \mu \mathrm{m}$; most of the data (345 runs) were collected with $\lambda=10.96 \mu \mathrm{m}$. The $e^{+}$ plasma was typically $1 \mathrm{~mm}$ in radius, and the laser beam waist in the mixing region was about $2 \mathrm{~mm}$ with a typical peak intensity of $160 \mathrm{~W} \mathrm{~cm}^{-2}$ at $10 \mathrm{~W}$ power.

The path of the laser beam through the interaction region was well constrained mechanically. Parallel displacements of the laser beam $( \pm 1.5 \mathrm{~mm}$ each side of the nominal central position) caused partial occlusion, and stability was assured by optimizing and continuously monitoring the laser power transmitted through the apparatus. In order to exclude suboptimal overlap, the $\overline{\mathrm{H}}$ production rate was measured with the laser displaced $1 \mathrm{~mm}$ in each of the four directions, and no significant difference was observed. To ensure the same mixing environment, the laser beam was chopped at a frequency of $25 \mathrm{~Hz}$, recording the state of the laser (on or off) simultaneously with the annihilation events. In this way, 156160 annihilation events were collected in 345 cold mix runs, each with a duration of $50 \mathrm{~s}$.

The time distribution of the $\bar{p}$ annihilation vertices on the trap wall for all of these runs is presented in Fig. 1. This distribution is due mainly to $\overline{\mathrm{H}}$ annihilating on the trap wall with a background due to $\bar{p}$ annihilating on the residual gas atoms in the trap center [19]. The curve can be fitted with two exponentials (not shown in the figure), having time constants of $(11.2 \pm 1.4)$ and $(76.8 \pm 8.3) \mathrm{s}$, respectively. This time behavior is characteristic for our $\overline{\mathrm{H}}$ formation

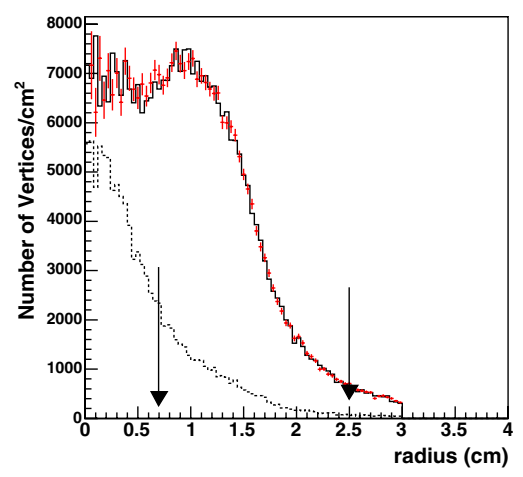

FIG. 2 (color online). Radial vertex distribution for laser off (line) and laser on (crosses) events. The arrows indicate the region considered in Table I. The dashed histogram is the normalized background measured from the hot mix runs.

procedure, where different cooling regimes have been observed [7].

The radial vertex density, shown in Fig. 2, represents the $\bar{p}$ annihilation vertex position as reconstructed by the hits of the charged mesons in the two silicon strip layers of the detector. The antiatoms annihilating on the trap wall at a radius of $1.25 \mathrm{~cm}$ give rise to a peak in the distribution, smeared by the resolution of the vertex algorithm $(1.8 \mathrm{~mm}$ along the trap axis and $3.5 \mathrm{~mm}$ in the transverse direction) [4]. By fitting this distribution with the sum of the measured background from the hot mix runs (see Fig. 2) and the vertex density for pure $\overline{\mathrm{H}}$ annihilation from our Monte Carlo simulation [19], we obtain the fraction of $\overline{\mathrm{H}}$ events of $(67 \pm 4) \%$. Taking into account the efficiencies as in Ref. [19], in total we produced about $7 \times 10^{5} \overline{\mathrm{H}}$, corresponding to an observed mean rate of $40 \mathrm{~s}^{-1}$, and an overall yield of $(20 \pm 4) \%$.

The cosine of the opening angle distribution, shown in Fig. 3 , represents the angle $\theta_{\gamma \gamma}$ between the two $511 \mathrm{keV} \gamma$ rays recorded in time coincidence with the charged-particle hits, as seen from the charged-particle vertex. The clear excess at $\cos \left(\theta_{\gamma \gamma}\right)=-1$ (corresponding to back-to-back emission of the two $\gamma$ from the $e^{+}-e^{-}$annihilation) is

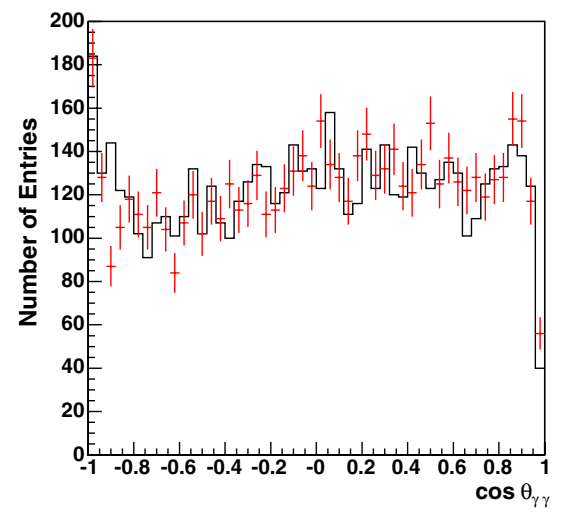

FIG. 3 (color online). Opening angle distributions for laser off (line) and laser on (crosses) events. 
proof of the presence of antihydrogen [1,19]. These distributions show no evidence for laser-stimulated $\overline{\mathrm{H}}$ formation. This conclusion does not depend upon variations in the temporal or spatial cuts applied to the vertex and angle distributions.

To be more quantitative, we consider the number of vertices over the entire $50 \mathrm{~s}$ mixing period and in the subintervals $1<t \leq 10 \mathrm{~s}$ and $10<t<50 \mathrm{~s}$, a demarcation prompted by the two time components present in our data (the results are insensitive to small variations of the widths of these intervals). In this analysis, we also use a subsample of particularly well reconstructed vertices, using the quality index from the vertex algorithm; since this index is calculated on the basis of statistical estimators only, the vertex quality selection has no physical bias [4].

The number of vertices recorded in different time windows is listed in Table I. A radial cut from $0.7<r<$ $2.5 \mathrm{~cm}$ (see Fig. 2) was applied to these data. Although this cut tends to suppress the background which is mainly in the trap center, similar results are obtained without radial cuts. The small effect that we observe from Table I is a $2.2 \sigma$ deficit of laser on events in the interval $10<t<$ $50 \mathrm{~s}$ (vertex quality applied).

Since in the region $0.7<r<2.5$ the signal and background percentages are $(87 \pm 4) \%$ and $(13 \pm 4) \%$, respectively, from Table I we find $90 \%$ C.L. upper limits of 3.8\% for the enhancement in the $[1,10] \mathrm{s}$ time interval (corresponding to $1.5 \mathrm{H} \mathrm{s}^{-1}$ from our observed mean $\overline{\mathrm{H}}$ rate of $40 \mathrm{~s}^{-1}$ ) and of $-3.6 \%$ for the deficit in the $[10,50] \mathrm{s}$ interval (corresponding to 1.4 ionized $\overline{\mathrm{H} \mathrm{s}}{ }^{-1}$ ). In the whole time interval $1<t<50 \mathrm{~s}$ and without vertex quality, we obtain a $90 \%$ C.L. upper limit for laser enhancement of $0.8 \%$, corresponding to $0.3 \overline{\mathrm{H} ~ s}{ }^{-1}$. The conclusion is not changed when the opening angle distributions are analyzed within the same time intervals, though the statistical significance is lower.

Investigations were also performed with different laser tunings in the range $\lambda=10.16 \mu \mathrm{m}$ to $\lambda=11.03 \mu \mathrm{m}$, in order to investigate whether a dependence on detuning could be observed. No statistically significant variations between laser on and off were found.

Considering the predicted enhancement factor of 3.5 due to the laser radiation with respect to the spontaneous radiative formation, and that $67 \%$ of the data are due to $\overline{\mathrm{H}}$ annihilations, it follows from Table I that, to observe a $5 \sigma$ difference between laser on and off annihilations, a radiative component $>1.5 \%$ of the total recombination rate is required [8]. In spite of this, we observe a null laser effect on $\overline{\mathrm{H}}$ production. Barring the unlikely possibility of a suboptimal overlap between the antiprotons and the laser beam, this result suggests that spontaneous two-body radiative formation in conditions of thermal equilibrium gives a negligible contribution to the $\overline{\mathrm{H}}$ formation in the ATHENA nested trap. Other results also indicate that $\overline{\mathrm{H}}$ formation does not occur under conditions of thermal equilibrium between the $\bar{p}$ and the $e^{+}[8,18,20]$, and it is
TABLE I. Integrated number of vertices in the arrowed region shown in Fig. 2 for different time windows $\left[t_{1}, t_{2}\right]$ in seconds.

\begin{tabular}{lcccc}
\hline \hline & Vertex & \multicolumn{3}{c}{ Time window (s) } \\
Run & quality & {$[1,50]$} & {$[1,10]$} & {$[10,50]$} \\
\hline Laser on & No & 59496 & 18457 & 41039 \\
Laser off & No & 59611 & 18353 & 41258 \\
On-off & & $-115 \pm 345$ & $104 \pm 192$ & $-219 \pm 287$ \\
Laser on & Yes & 34731 & 10938 & 23793 \\
Laser off & Yes & 35039 & 10760 & 24279 \\
On-off & & $-308 \pm 434$ & $178 \pm 241$ & $-486 \pm 219$ \\
\hline \hline
\end{tabular}

likely that three-body capture and collisional processes are the dominant mechanisms. Moreover, the laser ionization of the high- $n$ Rydberg states formed in this way could mask a small contribution from laser-induced radiative recombination.

In this scenario, to date most $\overline{\mathrm{H}}$ which has been detected has been produced in weakly bound Rydberg states. These results will provide a major impetus for the search for new, efficient methodologies for the production of cold groundstate antihydrogen.

*Present address: Max Planck Institute for Nuclear Physics, Postfach 103980, 69029 Heidelberg, Germany.

[1] M. Amoretti et al. (ATHENA Collaboration), Nature (London) 419, 456 (2002).

[2] G. Gabrielse et al., Phys. Rev. Lett. 89, 213401 (2002).

[3] G. Gabrielse et al., Phys. Lett. A 129, 38 (1988).

[4] M. Amoretti et al. (ATHENA Collaboration), Nucl. Instrum. Methods Phys. Res., Sect. A 518, 679 (2004).

[5] C. Regenfus, Nucl. Instrum. Methods Phys. Res., Sect. A 501, 65 (2003).

[6] M.C. Fujiwara et al. (ATHENA Collaboration), Phys. Rev. Lett. 92, 065005 (2004).

[7] M. Amoretti et al. (ATHENA Collaboration), Phys. Lett. B 590, 133 (2004).

[8] M. Amoretti et al. (ATHENA Collaboration), Phys. Lett. B 583, 59 (2004).

[9] M. Amoretti et al. (ATHENA Collaboration), Phys. Rev. Lett. 91, 055001 (2003); Phys. Plasmas 10, 3056 (2003).

[10] A. Müller and A. Wolf, Hyperfine Interact. 109, 233 (1997).

[11] M. Holzscheiter, M. Charlton, and M. M. Nieto, Phys. Rep. 402, 1 (2004).

[12] F. Robicheaux, Phys. Rev. A 70, 022510 (2004).

[13] P. O. Fedichev, Phys. Lett. A 226, 289 (1997).

[14] R. Neumann et al., Z. Phys. A 313, 253 (1983).

[15] U. Schramm et al., Phys. Rev. Lett. 67, 22 (1991).

[16] F. B. Yousif et al., Phys. Rev. Lett., 67, 26 (1991).

[17] A. Wolf, Hyperfine Interact. 76, 189 (1993).

[18] N. Madsen et al. (ATHENA Collaboration), Phys. Rev. Lett. 94, 033403 (2005).

[19] M. Amoretti et al. (ATHENA Collaboration), Phys. Lett. B 578, 23 (2004).

[20] G. Gabrielse et al., Phys. Rev. Lett. 93, 073401 (2004). 